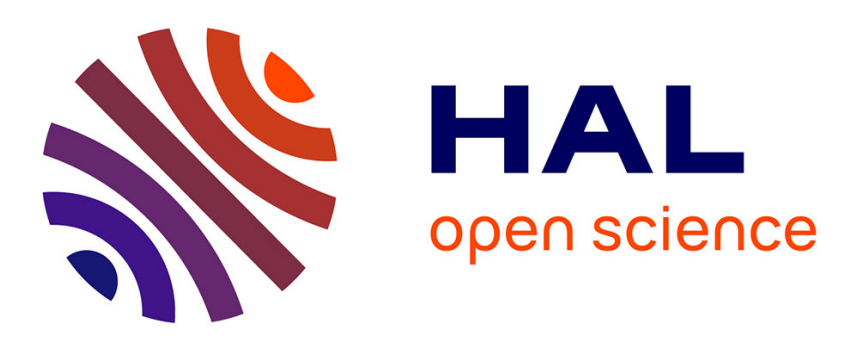

\title{
An Interfacial Friction Law for a Circular EHL Contact Under Free Sliding Oscillating Motion
}

\author{
Emmanuel Rigaud, D. Mazuyer, J. Cayer-Barrioz
}

\section{To cite this version:}

Emmanuel Rigaud, D. Mazuyer, J. Cayer-Barrioz. An Interfacial Friction Law for a Circular EHL Contact Under Free Sliding Oscillating Motion. Tribology Letters, 2013, 51 (3), pp.419-430. 10.1007/s11249-013-0177-z . hal-02068288

\section{HAL Id: hal-02068288 \\ https://hal.science/hal-02068288}

Submitted on 15 Mar 2019

HAL is a multi-disciplinary open access archive for the deposit and dissemination of scientific research documents, whether they are published or not. The documents may come from teaching and research institutions in France or abroad, or from public or private research centers.
L'archive ouverte pluridisciplinaire HAL, est destinée au dépôt et à la diffusion de documents scientifiques de niveau recherche, publiés ou non, émanant des établissements d'enseignement et de recherche français ou étrangers, des laboratoires publics ou privés. 
An interfacial friction law for a circular EHL contact under free sliding oscillating motion

E. Rigaud*, D. Mazuyer, J. Cayer-Barrioz

Laboratoire de Tribologie et Dynamique des Systèmes, UMR CNRS 5513

Ecole Centrale de Lyon, Université de Lyon

36, avenue Guy de Collongue, 69134 ECULLY cedex, France

Corresponding author: E. Rigaud

Tel: +33472186296

Fax: +33478433383

E-mail address: emmanuel.rigaud@ec-lyon.fr

19 pages

1 table

10 figures 


\begin{abstract}
The friction response of a lubricated interface under free sliding oscillating motion is investigated as a function of the contact pressure and the rheology of the lubricant in terms of viscosity and piezoviscosity. For loaded contacts, both velocity dependent friction, referred to as viscous damping, and friction independent of the instantaneous sliding velocity contribute to the energy dissipation. Viscous damping mainly corresponds to the dissipation in the lubricant meniscus surrounding the contact, while dissipation within the confined lubricated interface is mainly independent of the instantaneous sliding velocity. The friction coefficient independent of the instantaneous sliding velocity falls on a master curve for the wide range of tested operating conditions and lubricant rheological properties. The master curve is a logarithmic function of a dimensionless parameter corresponding to the ratio of the viscosity of the confined lubricant to the product of the pressure and a characteristic time. The physical meaning of this latter and the friction law are discussed considering the confined interface as a viscoelastic fluid or a non-Newtonian Eyring fluid.
\end{abstract}

\title{
Keywords
}

EHL Friction; Non-Newtonian Behaviour; Viscoelasticity; Friction master curve 


\section{Nomenclature}

a radius of the contact area

$c_{0} \quad$ viscous damping coefficient without contact

$h_{c} \quad$ central film thickness

$k \quad$ spring stiffness

m moving mass

$t_{c} \quad$ characteristic time

$x(t) \quad$ displacement response

$x_{\max } \quad$ initial displacement

E' reduced Young's modulus

$E(t) \quad$ energy decay

$E_{i}(t)$ energy dissipated by friction independent of the instantaneous sliding velocity

$E_{v}(t) \quad$ energy dissipated by viscous friction

$F_{n} \quad$ applied normal force

M dimensionless load parameter

P mean contact pressure

$P_{\max } \quad$ maximum contact pressure

$R_{x} \quad$ reduced radius of curvature

$S \quad$ Slide to roll ratio

$\bar{U} \quad$ dimensionless velocity

$U_{e} \quad$ entraining velocity

$U_{s} \quad$ sliding velocity

$\alpha \quad$ piezoviscous coefficient

$\bar{\alpha} \quad$ dimensionless piezoviscous coefficient

$\bar{\gamma} \quad$ mean shear rate

$\ddot{\gamma}_{\mathrm{E}} \quad$ effective shear rate

$\eta_{0} \quad$ dynamic viscosity at ambient pressure

$\eta(P)$ dynamic viscosity under contact pressure

$\bar{\eta} \quad$ dimensionless viscosity

$\mu \quad$ overall friction coefficient 


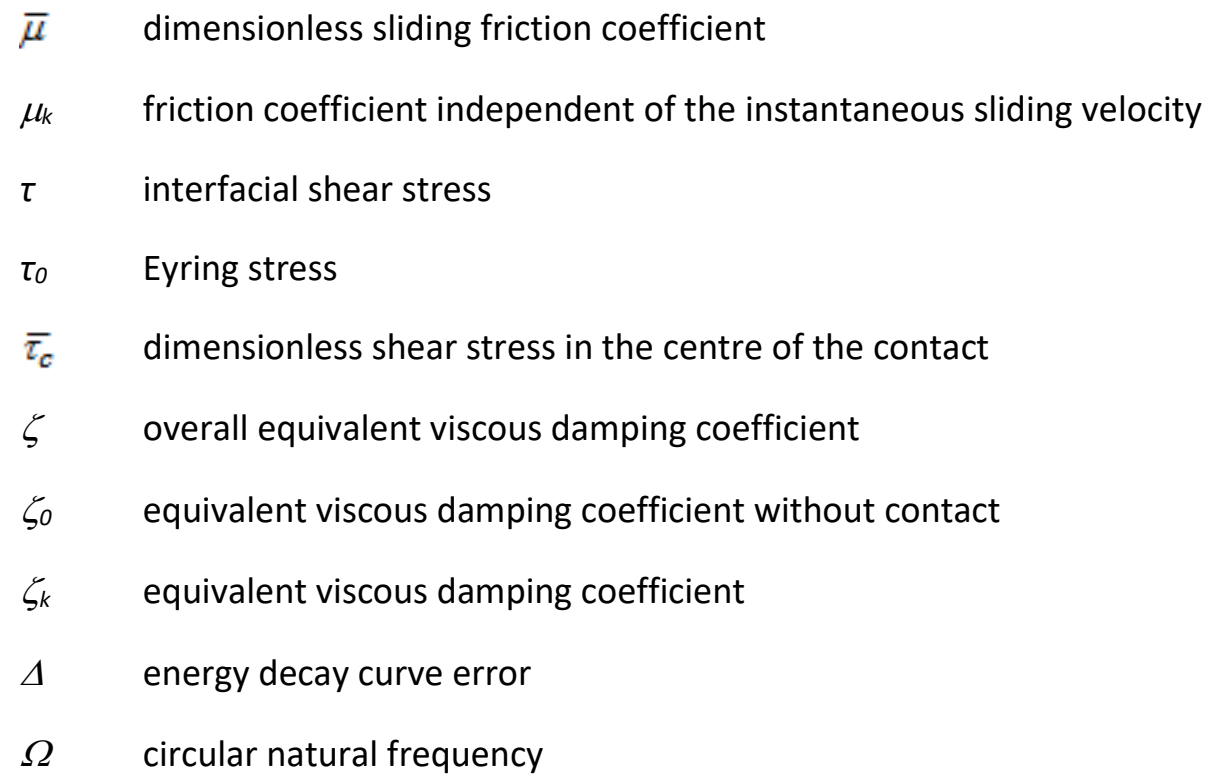

\section{1- Introduction}

In mechanical systems such as cam and followers, rolling elements bearings, gears and joints of living beings, many contacts between non-conformal surfaces operate in the elastohydrodynamic lubrication (EHL) regime. They are usually characterized by high contact pressure which leads to elastically deformed surfaces and thin lubricant films. For a point contact between smooth surfaces lubricated with piezoviscous Newtonian base oil under steady state rolling/sliding condition, the film thickness can be estimated from the widely used formulas derived by Hamrock and Downson [1] or Moes and Venner [2], as long as the slide to roll ratio remains low. An accurate prediction of friction induced by rolling and sliding through the contact is also needed to determine power losses and efficiency of machine components. Dissipation depends on the lubricant rheology which is strongly related to the high contact pressure in the confined interface. The lubricant viscosity under pressure can be estimated from the Barus equation [3] for moderate pressures. For higher pressures, more complex laws are proposed like the expression by Roelands [4] further written differently by Houpert [5]. Moreover, pressures, pressure gradients and shear rates encountered in EHL point contacts can be so high that sliding friction is not proportional to viscosity anymore: the lubricant becomes non Newtonian. Johnson and Tevaarwerk [6] have proposed a constitutive equation to model the shear of EHL films. The lubricant rheology is taken into account through a non linear Maxwell law as the sum of a linear element describing elastic strain and a nonlinear viscous element. The numerous models for the nonlinear viscous element are reviewed in [7]. A friction master curve is also proposed by Jacod et al. in the case of a high loaded smooth EHL rolling/sliding circular contact [8]. A dimensionless friction coefficient is computed assuming a lubricant that behaves according to an Eyring 
non-Newtonian model, for several lubricants and for different Eyring stresses ( $\left.\tau_{0}=4 \mathrm{MPa}, 6 \mathrm{MPa}, 8 \mathrm{MPa}\right)$, isothermal and stationary conditions, large values of the dimensionless load parameter $(50 \leq M \leq 1000)$ and slide to roll ratio such as $(0.1 \leq S \leq 2)$. After subtraction of the rolling friction contribution, the dimensionless friction due to sliding is considered. Jacod et al. suggested that it could be accurately approximated by the following formula:

$$
\bar{\mu}=\frac{P_{\max }}{\tau_{0}} \mu=\sinh ^{-1}\left(\frac{\overline{\tau_{c}}}{5}\right)
$$

$\bar{\tau}_{c}$ is a characteristic dimensionless shear stress in the centre of the contact, proportional to a dimensionless slip parameter function of the Eyring stress $\tau_{0}$, to the dimensionless viscosity for the maximum contact pressure, and to the reciprocal of the dimensionless central film thickness. In a paper related to the previous one, the dimensionless friction coefficient is computed assuming a lubricant that behaves according to the limiting shear stress model [9].

The laws predicting lubricant film thickness and friction presented above are established for steady-state conditions, while power losses may also occur in time varying operating conditions. For example, dynamic excitation can be superimposed to a mean static load. When the dynamic loading frequency coincides with the contact natural frequency, large variation of the contact normal force may appear [10-12] and the induced squeeze disrupts the film thickness [13] and the friction coefficient [14]. Moreover, many systems are intrinsically subjected to unsteady EHL conditions due to rolling, or sliding-rolling or pure sliding reciprocating motion. A large review of unsteady state non conformal EHL contact studies can be found in [15]. Researches often concern unsteady pure rolling or sliding of line and circular contacts. Most of the work is devoted to the film thickness analysis, while a minority is interested in friction. As this paper deals with friction in circular contacts under free oscillating sliding motion, we focus on the behaviour of EHL contact under reciprocating motion. The film formation is controlled by both wedging and squeezing actions. If the ratio of the stroke length to the contact radius is large enough, the maximum film thickness occurs at the stroke centre. Wang et al. [16] observed that it may be smaller than that corresponding to steady-state results for line contact. On the other hand, Nishikawa et al. [17, 18] observed that the maximum film thickness is almost the same as steady-state thickness for circular contacts under pure rolling or sliding, unless thermal effects modify the lubricant properties [17] or starvation occurs [18]. At stroke ends, the velocity reaches zero but the lubricant has no time to be squeezed out and a thick oil film is entrapped between the surfaces. The minimum film thickness at the reversal of entrainment velocity has been estimated by Hooke [19]. The central film thickness seems to be almost the same as that at stroke 
centre in sliding reciprocating motion, and contrary to rolling reciprocating motion [17]. Nevertheless, the oil film shape is changed and its thickness is thicker on the outlet side than on the inlet side. After the reversal of motion, Nishikawa et al. measured that the velocity of the entrapped film is about half the velocity of the sliding surface. The lubricant film is renewed from the inlet. The wedging and squeezing actions being out of phase generate a breathing corresponding to a periodic variation of the oil film shape $[17,20]$. According to Nishikawa et al. $[17,21]$, oil in the EHL conjunction does not behave as a viscous fluid during sinusoidal reciprocating sliding motion: the tangential force maintains an almost constant value in spite of large changes in the instantaneous velocity [17]. Furthermore, the value remains almost constant in a wide range of operating frequencies as long as full EHL is established [21].

In this paper, the friction in a lubricated sphere-plane contact under free sliding oscillating motion experiments is investigated. Experiments are performed on a dynamic oscillating tribometer which allows discrimination of the contribution independent of the sliding velocity from that depending on the sliding velocity. Several lubricants are used in order to vary the rheology of the confined fluid in terms of viscosity and piezoviscosity. The aim is to identify and discuss the interfacial friction law as a function of the rheology of the lubricant under pressure.

\section{2- Operating principle of the dynamic oscillating tribometer}

The dynamic oscillating tribometer [22] is a home-made friction measurement set-up which allows the evaluation of friction without measuring the tangential force at the interface. The friction properties of a sphere-plane sliding contact are deduced from the energy decay associated with the free response of a single degree-of-freedom damped oscillator corresponding to the sphere involved in the tribological system. The contribution independent of the instantaneous sliding velocity is discriminated from that depending on the sliding velocity (and referred to as equivalent viscous damping). The mechanical device principle is depicted in Fig. 1. The hemispherical pin is located at the extremity of a biblade that allows its oscillation according to the horizontal direction. The normal force is applied on the sphere-plane contact by micrometric vertical displacement of the frame and accommodation of an elastic blade. An electromagnet is used to generate the initial displacement of the hemispherical pin and to store potential energy in the biblade. When the electromagnetic force is removed, the free response of the oscillator is initiated until the pin stops due to the interfacial energy dissipation. Instantaneous displacement and velocity responses of the hemispherical pin are measured thanks to a laser vibrometer Polytec OFV-5000, that operates with a 
displacement resolution of $0.15 \mu \mathrm{m}$ and a velocity resolution equal to $0.015 \mu \mathrm{m} / \mathrm{s}$. Additional electrical contact resistance (ECR) measurement is performed.

The dynamic oscillating tribometer is modelled by a single-degree-of-freedom mechanical oscillator displayed in Fig. 2. The equation of motion is:

$$
m \ddot{x}+c_{0} \dot{x}+k x=-\mu(\dot{x}) \cdot F_{n}
$$

$x$ is the displacement of the system, $m$ is the moving mass, $c_{o}$ is the damping without contact, $k$ is the biblade stiffness, $F_{n}$ is the applied normal force and $\mu$ is the overall friction coefficient. The natural circular frequency $\Omega$ and the equivalent viscous damping coefficient $\zeta_{0}$ of the mechanical oscillator without contact or lubricant are defined as follows:

$$
\begin{aligned}
& \Omega=\sqrt{\frac{k}{m}} \\
& \zeta_{0}=\frac{c_{0}}{2 \sqrt{\mathrm{km}}}=\frac{c_{0}}{2 m \Omega}
\end{aligned}
$$

The following expression is assumed for the friction law describing the dissipation in the confined lubricated interface:

$$
\mu(\dot{x})=\mu_{k} \operatorname{sgn}(\dot{x})+2 \frac{\sqrt{k m}}{F_{n}} \zeta_{k} \dot{x} \quad(\dot{x} \neq 0)
$$

$\mu_{k}$ is the friction coefficient independent of the instantaneous sliding velocity. $\zeta_{k}$ is the equivalent viscous damping coefficient which reflects the dependence of the friction on the sliding velocity. The coefficients $\mu_{k}$ and $\zeta_{k}$ are assumed to be constant throughout the sliding velocity range experienced by the tribological system and independent of the instantaneous acceleration. Instantaneous potential, kinetic and total energy time responses are identified from displacement and velocity responses of the hemispherical pin:

$$
E(t)=\frac{1}{2} k x(t)^{2}+\frac{1}{2} m \dot{x}(t)^{2}
$$

The friction coefficient independent of the instantaneous sliding velocity $\mu_{k}$ and the equivalent viscous damping coefficient $\zeta=\zeta_{0}+\zeta_{k}$ are identified using the least squares method, by minimizing the indicator of the difference between the theoretical energy decay deduced from the equation of motion and the experimental energy decay:

$$
\Delta=\left(\frac{1}{N} \sum_{1}^{N}\left(E(t)_{t h}-E(t)_{\exp }\right)^{2}\right)^{1 / 2}
$$

The equivalent viscous damping coefficient $\zeta$ is decomposed into the contribution $\zeta_{0}$ corresponding to the viscous dissipation generated by the experimental device itself and the contribution $\zeta_{k}$ corresponding to 
the viscous interfacial dissipation. A preliminary experiment with neither contact, nor lubricant is performed in order to measure $\zeta_{0}$. Then, the equivalent viscous damping in the interface $\zeta_{k}$ is deduced from $\zeta_{k}=\zeta-\zeta_{0}$

\section{3- Materials and experimental conditions}

The contact is established between a $3 \mathrm{~mm}$ radius spherical pin and a flat surface. Solids are made of AISI 52100 steel. They are successively polished using diamond solutions with 6,3 and $1 \mu \mathrm{m}$ abrasive particles until their surfaces are mirror-polished with a roughness $R_{a}$ less than $5 \mathrm{~nm}$.

Three kinds of lubricants are used, i.e. polyalphaolefin blends (PAO), neutral solvent base oils (NS) and glycerol/water blends. This allows us to vary the rheology of the confined fluid in terms of viscosity at ambient pressure $\eta_{0}$ (from 0.03 to 3 Pa.s), and piezoviscosity $\alpha$ (between 5 and $2010^{-9} \mathrm{~Pa}^{-1}$ ). The mass fraction of water to obtain a given viscosity at ambient pressure for water/glycerol blends is determined from [23]. Fluid viscosities are measured at low shear rates using adequate capillary viscometers purchased from Schott@. Rheological properties of the lubricants are reported in Table 1.

The applied normal force can reach $0.6 \mathrm{~N}$ which corresponds to a maximum contact pressure up to $550 \mathrm{MPa}$ taking into account the geometry and elastic mechanical properties of solids. The moving mass $m$ is $29.2 \mathrm{~g}$ and the biblade stiffness $k$ is $945 \mathrm{~N} . \mathrm{m}^{-1}$ leading to a natural circular frequency $\Omega$ equal to 180 rad.s 1. The initial displacement is $0.56 \mathrm{~mm}$. The corresponding maximum sliding velocity is $0.1 \mathrm{~m} \cdot \mathrm{s}^{-1}$ and the corresponding initial energy is $0.15 \mathrm{~mJ}$. The ratio of the stroke length to the contact radius varies between 25 and 55 depending on the contact pressure. The acquisition frequency of the instantaneous displacement and velocity responses is equal to 1000 samples per second. Experiments are carried out at ambient temperature $\left(20^{\circ} \mathrm{C}\right)$ and humidity $(40 \% \mathrm{RH})$. Each experiment is reproduced at least four times. The successive responses are almost identical confirming that the dissipation during the experiment does not lead to a modification of the lubricant such as water intake in glycerol, nor to significant macroscopic thermal effects like a decrease of viscosity. The values indicated in the remaining of this paper correspond to mean values. Standard deviations remain lower than $5 \%$. Values of friction coefficients $\mu_{k}$ and $\zeta_{k}$ derived for different initial amplitudes (from $0.5 \mathrm{~mm}$ to $1 \mathrm{~mm}$ ) are almost the same. 


\section{4- Experimental results}

\subsection{Dissipation for unlubricated and lubricated unloaded contacts}

The damping characteristics of the experimental device itself have been identified from a preliminary experiment with neither contact, nor lubricant. Experimental velocity response and logarithm of the energy decay are displayed in Fig. $3 a$ and $3 \mathrm{~b}$ as a function of time. The velocity response is made of successive peaks with exponential decay. The corresponding logarithm of the energy decay is linear. This behaviour is typical of a purely viscous dissipation which can be modelled by an equivalent viscous damping $\zeta_{0}[22,24$, $25]$. There, the damping of the experimental device itself is very low: $\zeta_{0}=1.110^{-3}$. It can originate from several phenomena: inner damping inherent to the materials used, micro-sliding in the connections between mechanical parts, vibrations transmitted to the flat and acoustic radiation of surfaces vibrating in air.

Experiments without contact have also been performed for lubricated interfaces. A controlled volume of PAO blend with viscosity at ambient pressure $\eta_{0}$ varying from 0.03 to 3 Pa.s is introduced between the two surfaces. The distance between the two surfaces is close to zero but the contact remains unloaded. Fig. $3 a$ and $3 \mathrm{~b}$ also display the experimental velocity response and the energy decay as a function of time for several PAO blends. The curves are shifted for sake of clarity. The higher the viscosity, the less the number of oscillations before the motion stops. Once again, successive peaks of the velocity response highlight an exponential decay. The power dissipation for unloaded lubricated interfaces corresponds to a purely viscous one. The overall equivalent viscous damping $\zeta$ can be estimated and the value of $\zeta_{k}=\zeta-\zeta_{0}$ is plotted as a function of $\eta_{0}$, the viscosity of the lubricant meniscus at ambient pressure (see Fig 4). A linear variation of the equivalent viscous damping $\zeta_{k}$ with lubricant viscosity is observed. Viscous dissipation due to lubricant meniscus is much greater than that associated with the experimental device itself. $\zeta_{k}$ can reach values such as $510^{-2}$ for the viscosity $\eta_{0}=3$ Pa.s. It can be attributed to the deformation and displacement of the whole volume of fluid when the sphere oscillates over the plane. Additional experiments carried out with various volumes of lubricant confirm that $\zeta_{k}$ varies with the volume of fluid surrounding the contact: the smaller the volume of the moving meniscus, the lower $\zeta_{k}$.

\subsection{Dissipation in the confined interface}

A normal force varying from $0.1 \mathrm{~N}$ to $0.5 \mathrm{~N}$ has been applied to the contact lubricated with PAO blend 4 $\left(\eta_{0}=0.45\right.$ Pa.s). The corresponding maximum contact pressure (respectively average contact pressure) ranges from 305 to $520 \mathrm{MPa}$ (respectively from 204 to $347 \mathrm{MPa}$ ). Fig. 5a displays the velocity response and 
the corresponding electrical contact resistance (ECR). Fig. 5b displays the energy decay. The duration of the experiment before the signal extinction is less than $1 \mathrm{~s}$.

Whatever the applied normal force, we observe that ECR remains constant at a rather large value until the last oscillation when it suddenly drops. We assume that a lubricant film is entrained in the contact at an entraining velocity $U_{e}=U_{s} / 2$, where $U_{s}$ is the sliding velocity between surfaces at the stroke centre. At stroke ends, the sliding velocity decreases to zero. However, the lubricant has no time to be squeezed out due to the reversal of velocity and a thick oil film is entrapped between the surfaces, as previously observed and modelled [16-21]. The presence of this oil film allows us to explain the ECR data. At the end of motion, ECR reaches its lowest value only a few tens of ms after the complete stop.

The velocity responses show that the higher the applied normal force, the higher the dissipation and the less the number of oscillations before the motion stops. Unlike the unloaded contact response, it can be seen that successive peaks show a decay which is neither linear nor exponential. It means that the dissipation is not purely viscous although the contact is lubricated by a film that completely separates the surfaces, as previously observed by Nishikawa et al. for similar sinusoidal reciprocating sliding motion [17, 21]. Viscous damping and friction independent of the instantaneous sliding velocity coexist. The coefficients $\mu_{k}$ and $\zeta_{k}$ of the interfacial friction law defined in Eq. (5) have been identified from the methodology described in detail in [22] and summarized in the previous section (corresponding values are a part of results presented Fig. 7). The very low value of the indicator $\Delta$ (see Eq. 7) indicates that the proposed interfacial friction law accurately accounts for the dissipation in the confined lubricated interface. The part of initial energy dissipated in the friction independent of the instantaneous sliding velocity and the part of initial energy dissipated in the viscous damping can be discriminated as follows:

$$
E=E_{i}+E_{v}=\mu_{k} \cdot F_{n} \int \operatorname{sign}(\dot{x}) \dot{x}(t) d t+\zeta_{k} 2 \sqrt{k m} \int \dot{x}(t)^{2} d t
$$

Fig. 6 confirms that the energy is dissipated in viscous damping for unloaded contact, while the part of initial energy dissipated in friction independent of the instantaneous sliding velocity increases continuously until $85 \%$ for the largest contact pressure.

Similar experiments have been carried out for loaded contacts lubricated by fluids with various viscosities and piezoviscosities, such as reported in Table 1. For the lowest viscosity blends, ECR analysis shows that intermittent solid asperities interactions occur. Therefore, the experiments whose results are discussed in the remaining of this paper correspond to those for which ECR remains constant at a large value during motion, consistently with lubricant film persistence between the surfaces. 
Figure 7 displays the equivalent viscous damping coefficient $\zeta_{k}$ (Fig. 7a) and the friction coefficient $\mu_{k}$ (Fig. 7b) as a function of the normal force for piezoviscous PAO blends 3 to 6 , while Fig 8 displays results obtained for isoviscous glycerol/water blends 4 to 6 .

The coefficient $\zeta_{k}$ varies between 0.005 and 0.02 according to the lubricant viscosity. Fig. 7a and 8a show that $\zeta_{k}$ is almost independent of the applied normal force for both piezoviscous and isoviscous lubricants. Its value remains consistent with that obtained at zero normal force for a similar volume of fluid. This tends to show that the viscous damping is mainly associated with the energy dissipated in the area around the contact.

Consequently we may consider that the dissipation within the confined lubricated interface is mainly independent of the instantaneous sliding velocity. Regarding the coefficient $\mu_{k}$ which arises when the normal force is applied, it increases with the viscosity of the lubricant at ambient pressure. However, two tendencies can be distinguished according to the piezoviscosity (see Fig. $7 \mathrm{~b}$ and $8 \mathrm{~b}$ ): when increasing the applied normal force, $\mu_{k}$ continuously increases up to 0.05 for piezoviscous lubricant, while it remains rather constant for isoviscous lubricant, at a value which is less than 0.01 for glycerol/water blends. This seems to indicate that $\mu_{k}$ depends on the viscosity under pressure $\eta(P)$. The latter has been calculated taking account of the piezoviscosity values reported in Table 1. Fig. 9 clearly states that the coefficient $\mu_{k}$ logarithmically increases as $\eta(P)$ goes up.

\subsection{Interfacial friction master curve}

Since each curve $\mu_{k}=f(\eta(P))$ plotted in Fig. 9 seems to be shifted from one another, the following dimensionless parameters are introduced as a function of the operating conditions and the rheological properties of the lubricant, in order to propose an interfacial friction master curve:

$$
\begin{cases}\bar{U}=\frac{\eta_{0} U_{e}}{E^{\prime} R_{x}} & \text { dimensionless velocity } \\ \bar{\alpha}=\alpha P & \text { dimensionless piezoviscous coefficient } \\ \bar{\eta}=\frac{\eta(P)}{\eta_{0}} & \text { dimensionless viscosity }\end{cases}
$$

$E^{\prime}$ is the reduced Young's modulus, $R_{x}$ is the reduced radius of curvature, $P$ is the mean contact pressure and $U_{e}$ is the entraining velocity $\left(U_{e}=U_{s} / 2\right.$ for a sliding contact, where $U_{s}$ is the sliding velocity).

A characteristic time $t_{c}$ is defined from the dimensionless parameter $\eta(P) /\left(t_{c} . P\right)$ : 


$$
\frac{1}{t_{c}} \frac{\eta(P)}{P}=A \bar{U}^{m} \bar{\alpha}^{n} \bar{\eta}
$$

leading to:

$$
t_{c}=A^{-1} \eta_{0}{ }^{1-m} \alpha^{-n} P^{-n-1}\left(\frac{2 E^{\prime} R_{x}}{U_{s}}\right)^{m}
$$

All data from Fig. 9 can be rescaled with:

$$
A=1800, \quad m=\frac{1}{3}, \quad n=-\frac{8}{15}
$$

That is to say:

$$
t_{c}=\frac{1}{1800} \eta_{0}{ }^{2 / 3} \alpha^{8 / 15} P^{-7 / 15}\left(\frac{2 E^{\prime} R_{x}}{U_{s}}\right)^{1 / 3}
$$

Fig. 10 shows that all data fall on a master curve as a function $\eta(P) /\left(t_{c} . P\right)$. Whatever the lubricant rheology and the operating conditions, the following interfacial friction law can be proposed:

$$
\mu_{k}=0.035 \log \left(\frac{1}{t_{c}} \frac{\eta(P)}{P}\right) \quad \text { or } \quad \mu_{k}=0.015 \ln \left(\frac{1}{t_{c}} \frac{\eta(P)}{P}\right)
$$

The estimate of the coefficient of determination for the proposed linear regression, plotted in dashed line in Fig. 10, leads to a value $\mathrm{R}^{2}=0.985$. Nevertheless, a slight deviation of experimental results with respect to the master curve is observed for isoviscous water/glycerol blends corresponding to low values of $\eta(P) /\left(t_{c} . P\right)$. Taking account of the mean contact pressure and the maximum sliding velocity, the characteristic time $t_{c}$ ranges from $2.5 \times 10^{-9}$ to $3 \times 10^{-8} \mathrm{~s}$ for the studied lubricants. However, its physical meaning needs to be discussed. In addition, one can wonder why the interfacial friction law links the friction coefficient independent of the instantaneous sliding velocity to the lubricant rheology.

\section{5- Discussion}

\subsection{Relation between the characteristic time $t_{c}$ and the shear rate of the lubricated interface $\gamma$}

Assuming a linear velocity distribution through the film thickness, the mean shear rate of the lubricated interface is introduced as follows [6]:

$$
\dot{\gamma}=\frac{U_{s}}{h_{c}}
$$

where $h_{c}$ is the central film thickness and $U_{s}$ is the sliding velocity.

Since the ratio of the stroke length to the contact radius is large enough, we first assume that the spatial distribution of film thickness at the stroke centre is close to that observed in stationary conditions. As a second approximation, the central film thickness at the stroke end is supposed to be as the same order of magnitude as that at stroke centre, as previously observed for circular contact under sliding [17]. The mean 
central film thickness $h_{c}$ can be related to the entraining velocity at the stroke centre by the HamrockDowson equation [1]:

$$
h_{c}=2.69 R_{x}(1-0.61 \exp (-0.73 k)) \cdot\left(\frac{\eta_{0} U_{e}}{E^{\prime} R_{x}}\right)^{2 / 3} \cdot\left(\alpha E^{\prime}\right)^{8 / 15} \cdot\left(\frac{F_{n}}{E^{\prime} R_{x}{ }^{2}}\right)^{-1 / 15}
$$

where $k$ is the ellipticity parameter.

Considering the sphere plane contact $(k=1)$, the entraining velocity for a sliding contact $\left(U_{e}=U_{s} / 2\right)$ and the mean contact pressure as a function of the normal force [26]:

$$
h_{c}=1.6403 R_{x}\left(\frac{\eta_{0} U_{s}}{2 E^{\prime} R_{x}}\right)^{2 / 3} \cdot\left(\alpha E^{\prime}\right)^{8 / 15} \cdot\left(\frac{\pi P}{E^{\prime}}\right)^{-1 / 5}
$$

and $\frac{1}{\dot{\gamma}}=0.652 \eta_{0}{ }^{2 / 3} \cdot \alpha^{8 / 15} \cdot\left(\frac{2 R_{x}}{U_{s}}\right)^{1 / 3} P^{-1 / 5} \cdot E^{\prime 1 / 15}$

The film thickness estimate is not accurate because of the previous assumptions and the large sliding/rolling ratio. In addition, for a few of the performed experiences, the dimensionless load parameter is low $(M \leq 10)$ and the more complex Moes-Venner formula would have been more appropriate [2]. Nevertheless, taking account of Eq. (12) and (17), a simple analytical relation can be established between the characteristic time $t_{c}$ and the mean shear rate experienced by the lubricated interface $\mathscr{\gamma}$ :

$$
t_{c} \dot{\gamma}=8.510^{-4}\left(\frac{E^{\prime}}{P}\right)^{4 / 15}
$$

The influence of the contact pressure on the dimensionless parameter $t_{c}^{i} /$ is rather low due to the small value of the corresponding power function coefficient. Therefore, $t_{c}^{i} /$ can be approximated as follows:

$$
t_{c} \dot{\gamma} \approx 5.110^{-3}
$$

\subsection{Modelling of the lubricant using a viscoelastic fluid}

The interfacial friction law master curve can be discussed in terms of rheological behaviour of the confined lubricant. First, the friction independent of the instantaneous sliding velocity could be explained by an elastic contribution to dissipation. Hypothesis of a confined lubricant which behaves like a linear viscoelastic material is discussed using Deborah number $D$. This one is defined as the ratio of the relaxation time for the confined lubricant $(\eta(P) / G)$ to its dwell time through the contact zone $\left(2 a / U_{s}\right)[6]$ :

$$
D=\frac{\eta(P)}{G} \frac{U_{s}}{2 a}
$$

with $G=\mu_{k} P \frac{h_{c}}{a}$ 
$G$ is the apparent elastic shear modulus of the lubricant and $a$ is the radius of the contact area. Elastic response of the lubricant film is associated with large values of $D$, while negligible elastic effects correspond to small values of $D$. Taking account of Eq. (14) and (18), $D$ can be related to the mean shear rate $\gamma$ or the characteristic time $t_{c}$ :

$$
D=\frac{\eta(P)}{P} \frac{\dot{\gamma}}{2 \mu_{k}} \approx \frac{2.0510^{-3}}{\mu_{k} t_{c}} \frac{\eta(P)}{P}
$$

For isoviscous lubricants, $D$ is almost constant at a value close to 0.4 . For piezoviscous lubricants, $D$ rises from 0.3 to 4.5 with viscosity under pressure. A Deborah number of the order of the unity confirms the existence of an elastic contribution to dissipation that could explain the friction part independent of the instantaneous sliding velocity. Its evolution with viscosity under pressure shows that the confined lubricant behaviour is more and more "solid-like" rather than "liquid-like". Nevertheless, one could expect higher values of $D$ in order to confirm the predominantly elastic behaviour of the confined interface. For such values of $D$, a viscous contribution to the friction in the confined interface was also expected, while the equivalent viscous damping coefficient is almost constant whatever the contact pressure, at a value consistent with that obtained for unloaded contact. This result may be due to the numerous assumptions made in the above discussion. As a first approximation, the Deborah number is evaluated assuming a linear velocity distribution through the film thickness and uniform values of the lubricant parameters throughout the contact, while sliding between surfaces may generate thermal effects and heterogeneous properties of the lubricant through the interface [16]. Moreover, the dwell time of the lubricant through the contact zone is less than $1 \mathrm{~ms}$, so that its properties have not reached equilibrium conditions. Then, the periodic variation of the central film thickness and global film shape are neglected and an approximated constant central film thickness is used. Finally, a linear elastic model is an additional assumption, while large deformation of the interface may be associated with non linear effects.

\subsection{Modelling of the lubricant using a non-Newtonian Eyring fluid}

Another approach would consist in considering that the confined lubricant behaves like a non-newtonian fluid whose viscous flow is described by Eyring's model. The interfacial shear stress $\tau$ can be written as:

$$
\frac{\tau}{\tau_{0}}=\sinh ^{-1}\left(\frac{\eta(P) \dot{\gamma_{E}}}{\tau_{0}}\right)=\ln \left(\frac{\eta(P) \dot{\gamma}_{E}}{\tau_{0}}+\sqrt{1+\left(\frac{\eta(P) \dot{\gamma}_{E}}{\tau_{0}}\right)^{2}}\right)
$$


where $\tau_{0}$ is the Eyring stress above which the lubricant is non-newtonian and ${ }^{b}{ }_{\mathrm{E}}$ is the effective shear rate of the fluid. Considering that the friction response of the confined lubricant raises from its viscous shearing, the friction coefficient $\mu_{k}$ is given by:

$$
\mu_{k}=\frac{\tau}{P}=\frac{\tau_{0}}{P} \sinh ^{-1}\left(\frac{\eta(P) \dot{\gamma}_{E}}{\tau_{0}}\right)=\frac{\tau_{0}}{P} \ln \left(\frac{\eta(P) \dot{\gamma}_{E}}{\tau_{0}}+\sqrt{1+\left(\frac{\eta(P) \dot{\gamma_{E}}}{\tau_{0}}\right)^{2}}\right)
$$

For viscous stress $\eta(P)^{n} \gamma_{\mathrm{E}}$ much higher than $\tau_{0}$, Eq. (24) becomes:

$$
\mu_{k} \approx \frac{\tau_{0}}{P} \ln \left(\frac{2 \eta(P) \dot{\gamma}_{E}}{\tau_{0}}\right)
$$

Thus, by comparing Eq. (25) to Eq. (13), a simple analytical relation can be established between the Eyring stress and the mean contact pressure:

$$
\frac{\tau_{0}}{P}=0.015
$$

$\tau_{0}$ varies between 2.4 and $5.5 \mathrm{Mpa}$ in the range of experimental conditions considered.

The characteristic time $t_{c}$ can also be related to the effective shear rate $\hat{\gamma}_{\mathrm{E}}$ :

$$
t_{c} \dot{\gamma}_{E}=\frac{\tau_{0}}{2 P}=7.510^{-3}
$$

The Eyring's model of viscosity assumes the flow as a thermally activated process that involves the displacement of a rheological unit [27], whose volume is proportional to the reciprocal of $\tau_{0}$. Therefore, Eq. (26) suggests that the higher the pressure, the smaller the activated volume. Assuming a dependence of the size of the rheological unit with pressure is realistic although this result was not foreseen by Eyring's theory.

Moreover, it can be worth noting that effective shear rate $\hat{\gamma}_{\mathrm{E}}$ defined Eq. (27) is close to the mean shear rate $\hat{\gamma}$ defined Eq. (19) from the mean central film thickness estimate. Expression of ${ }^{\not}{ }_{\mathrm{E}}$ as a function of rheological properties of the lubricant and operating conditions can be deduced from Eq. (12) and (27):

$$
\dot{\gamma}_{E}=13.5 \eta_{0}^{-2 / 3} \alpha^{-8 / 15} P^{7 / 15}\left(\frac{U_{s}}{2 E^{\prime} R_{x}}\right)^{1 / 3}
$$

$\hat{\gamma}_{\mathrm{E}}$ varies between $2.5 \times 10^{5} \mathrm{~s}^{-1}$ and $3 \times 10^{6} \mathrm{~s}^{-1}$ in the range of experimental conditions considered. The friction coefficient $\mu_{k}$ can be calculated as a function of $\eta(P) /\left(t_{c} . P\right)$ by combining Eq. (24) and (27):

$$
\mu_{k}=0.015 \sinh ^{-1}\left(\frac{1}{2 t_{c}} \frac{\eta(P)}{P}\right)=0.015 \ln \left(\frac{1}{2 t_{c}} \frac{\eta(P)}{P}+\sqrt{1+\left(\frac{1}{2 t_{c}} \frac{\eta(P)}{P}\right)^{2}}\right)
$$

The computed curve has been plotted in Fig. 10, using continuous line. It confirms that the friction behaviour is described by a master curve for all the lubricants. It shows a perfect agreement with the 
experimental results obtained with the piezo-viscous lubricants. This justifies a posteriori that the viscous stress $\eta(P)^{n} \mathrm{E}$ is much higher than the critical stress $\tau_{0}$ for Neutral Solvant base oils and PAO blends. A slight deviation of experimental results with respect to the master curve is observed for isoviscous water/glycerol blends. This discrepancy may have three origins:

- the Eyring's theory is not suitable to describe the rheology of the confined isoviscous lubricants, - calculation are made by assuming the piezoviscous coefficient for water/glycerol blends corresponds to piezoviscous coefficient for pure glycerol reported in Table 1,

- Eq. (26) that links the critical shear stress $\tau_{0}$ to mean contact pressure $P$ has been derived from piezoviscous lubricants with high viscous stress and cannot be extended to isoviscous fluids.

The friction law proposed by Jacod et al. (Eq. (1)) has been compared with the proposed interfacial friction law corresponding to Eq. (29). As a first difference, Jacod et al. assumed that the shear of the lubricant film in the centre of the contact is the predominant source of friction. So, the dimensionless sliding friction coefficient and viscosity were computed for the maximum contact pressure instead of the mean contact pressure. Furthermore, Jacod et al. considered a constant Eyring stress $\tau_{0}$, while we could establish a linear relationship between $\tau_{0}$ and the mean contact pressure $P$ (see Eq. (26)). Taking account of this relation, we found that prediction of friction coefficient with Jacod's formula leads to lower values than the ones measured: for piezoviscous lubricants and applied load such as $(M \geq 5)$, sliding friction coefficient predicted with Eq. (1) is only about $70 \%$ of the measured value and, for isoviscous lubricant corresponding to low values of $\eta(P) /\left(t_{c} . P\right)$ or $\bar{\tau}_{c}$, it is close to zero. These discrepancies can be attributed to the difference between the test conditions explored in our experiments and in Jacod's simulations. The range for dimensionless load parameter is $(0.5 \leq M \leq 30)$ instead of $(50 \leq M \leq 1000)$. The corresponding range for dimensionless shear stress is $\left(10^{-1} \leq \bar{\tau}_{c} \leq 10^{3}\right)$ instead of $\left(10^{0} \leq \bar{\tau}_{c} \leq 10^{20}\right)$ also corresponding to a range $\left(1.5 \leq \eta(P) /\left(t_{c} \cdot P\right) \leq 150\right)$. It is noteworthy that not only the load conditions considered to establish Eq. (1) and Eq. (29) are different, but also the friction coefficient corresponds to oscillating motion instead of stationary velocity. It is therefore justified to introduce a new interfacial friction law dedicated to these operating conditions.

\section{6- Conclusions}

Friction in a lubricated interface under free oscillating motion was investigated using an experimental methodology able to discriminate the viscous damping and the contribution independent of the instantaneous sliding velocity. The influence of rheological properties of the lubricant has been investigated 
in terms of viscosity and piezoviscosity. First, we showed that the dissipation for unloaded contact is purely viscous and is related to the lubricant viscosity at ambient pressure, as well as the volume of the meniscus that is deformed and displaced during the oscillating motion. Second, we showed that the energy dissipation for loaded contacts is associated with both viscous damping and friction independent of the instantaneous sliding velocity. The rate of energy dissipated through the latter rapidly grows with an increasing normal force. Whatever the mean contact pressure, the viscous damping coefficient $\zeta_{k}$ remains almost constant at a value consistent with that obtained at zero normal force. This leads us to consider that the viscous damping is mainly associated with the energy dissipated in the area around the contact and the friction dissipation within the confined lubricated interface is mainly attributed to the friction independent of the instantaneous sliding velocity. Whatever the lubricant, a logarithmic evolution of the corresponding friction coefficient $\mu_{k}$ as a function of the viscosity of the lubricant under pressure is observed. Finally, a characteristic time $t_{c}$ is introduced as a function of lubricant rheology and operating conditions. We demonstrate that friction coefficient $\mu_{k}$ of all the tested lubricants falls on a master curve as a logarithmic function of $\eta(P) /\left(t_{c} . P\right)$. The proposed equation should be useful for engineering use to predict sliding friction as a function of operating conditions.

The existence of an elastic contribution to dissipation in the confined interface could explain why a part of friction is independent of the instantaneous sliding velocity, as confirmed by the Deborah number estimate and its growing with contact pressure. Nevertheless, the absence of an additional viscous contribution is surprising. On the other hand, considering a non-Newtonian Eyring fluid in the confined interface allows us to predict the existence of friction coefficient $\mu_{k}$ master curve for both isoviscous and piezoviscous lubricants. In this framework, the Eyring stress $\tau_{0}$ is proportional to the contact pressure and the characteristic time $t_{c}$. is proportional to the reciprocal of the effective shear rate.

In addition to this work, methods for identifying friction parameters in forced oscillators will be implemented in order to compare results with the interfacial friction law identified under free sliding oscillating motion. As the periodic variation of the lubricant film shape appears to play a significant role on the friction characteristics, another step to deepen our analysis would consist in varying the motion frequency while simultaneously visualizing the contact by means of interferometric test apparatus and measuring velocity dependent and velocity independent friction contributions. 


\section{7- Aknowledgments}

The authors would like to thank Dr F. Brémond for helpful discussions. They are indebted to the institute Carnot Ingénierie@Lyon (I@L) for its support and funding.

\section{8- References}

[1] Hamrock, B.J., Dowson, D.: Isothermal elastohydrodynamic lubrication of point contacts. Part 1: theoretical formulations", ASME Journal of lubrication technology 98, 223-229 (1976).

[2] Nijenbanning, G., Venner, C.H., Moes, H.: Film thickness in elastohydrodynamically lubricated elliptic contacts. Wear 176(2), 217-229 (1994).

[3] Barus, C.: Isothermals, isopiestics and isometrics relative to viscosity. American Journal of Science 45, 87-96 (1893).

[4] Roelands, C.J.: Correlational aspect of viscosity-temperature-pressure relationships of lubricating oils. PhD thesis, Delft University of Technology, The Netherlands (1966).

[5] Houpert, L.: New results of traction force calculation in elastohydrodynamic contacts. ASME Journal of Tribology 107, 241-248 (1985).

[6] Johnson, K.L., Tevaarwerk, J.L.: Shear behaviour of elastohydrodynamics oil films. Proceedings of the Royal Society A, 356, 215-236 (1977).

[7] Bou-Chakra, E., Cayer-Barrioz, J., Mazuyer, D.: A non-Newtonian model based on Ree-Eyring theory and surface effect to predict friction in elastohydrodynamic lubrication. Tribology International 43, 1674-1682 (2010).

[8] Jacod, B., Venner C.H., Lugt, P.M.: A generalized traction curve for EHL contacts. ASME Journal of Tribology 123, 248-253 (2001).

[9] Jacod, B., Venner, C.H., Lugt, P.M.: Extension of the friction mastercurve to limiting shear stress models. ASME Journal of Tribology 125, 739-746 (2003).

[10] Nayak, R.: Contact vibrations. Journal of Sound and Vibration 22(3), 297-322 (1972).

[11] Rigaud, E., Perret-Liaudet, J.: Experiments and numerical results on nonlinear vibrations of an impacting hertzian contact. Part 1: harmonic excitation. Journal of Sound and Vibration 265(2), 289-307 (2003).

[12] Perret-Liaudet, J., Rigaud, E.: Experiments and numerical results on nonlinear vibrations of an impacting hertzian contact. Part 2: random excitation. Journal of Sound and Vibration 265(2), 309-327 (2003) 
[13] Nishikawa, H., Handa, K., Teshima, K. Matsuda, K., Kaneta, M.: Behavior of EHL films in cyclic squeeze motion. JSME International journal, serie C 38(3), 577-585 (1995).

[14] Hess, D., Soom, A.: Normal vibrations and friction under harmonic loads: Part 1: Hertzian contact. ASME Journal of Tribology 113, 80-86 (1991).

[15] Ciulli, E.: Non-steady state non-conformal contacts: friction and film thickness studies. Meccanica 44, 409-425 (2009).

[16] Wang, J., Kaneta, M., Yang, P.: Numerical analysis of TEHL line contact problem under reciprocating motion. Tribology International 38, 165-178 (2005).

[17] Nishikawa, H., Handa, K., Kaneta, M.: Behavior of EHL films in reciprocating motion. JSME International journal, serie C 38(3), 558-567 (1995).

[18] Wang, J., Hashimoto, T., Nishikawa, H., Kaneta, M.: Pure rolling elastohydrodynamic lubrication of short stroke reciprocating motion. Tribology International 38, 1013-1021 (2005).

[19] Hooke, C.J.: The minimum film thickness in line contacts during reversal of entrainment. Journal of tribology 115(1), 191-199 (1993).

[20] Petrousevitch, A.I., Kodnir, D.S., Salukvadze, R.G., Bakashvili, D.L., Schwarzman, V.S.: The investigation of oil film thickness in lubricated ball-race rolling contact. Wear 19(4), 369-389 (1972).

[21] Nishikawa, H., Kaneta, M.: Traction in EHL under pure sliding reciprocation with cyclic impact loading. JSME International journal, serie C 38(3), 568-576 (1995).

[22] Rigaud, E., Perret-Liaudet, J., Belin, M., Martin, J.M.: A dynamical tribotest discriminating friction and viscous damping. Tribology International 43(1-2), 320-329 (2010).

[23] Shankar, P., Kumar, M.: Experimental determination of the kinematic viscosity of glycerol-water mixtures. Proceedings of the Royal Society: Mathematical and Physical Sciences 444, N¹922, 573-581 (1994).

[24] Rayleigh, J.: The theory of sound. Vol. 1, reprinted by Dover, New York, 1945, 35-40 (1877).

[25] Belin, M., Kakizawa, M., Rigaud, E.; Martin, J.M.: Dual characterization of boundary friction thanks to the harmonic tribometer: identification of viscous and solid friction contributions. Journal of physics: conference series 258(1) (2010).

[26] Stachowiak, G.W., Batchelor, A.W.: Engineering tribology. Butterworth-Heinemann, Boston, $3^{\text {rd }}$ edition (2005).

[27] Eyring, H.: Viscosity, Plasticity, and Diffusion as Examples of Absolute Reaction Rates. Journal of Chemical Physics 4, 283-291 (1936). 


\section{List of tables and figures}

Table 1. Rheological properties of the lubricants.

Fig. 1. Dynamic oscillating tribometer.

Fig. 2. Single degree of freedom model.

Fig. 3. Velocity time response and energy decay for unloaded contact and viscosity of the lubricant meniscus varying from 0 (no lubricant) to 3.10 Pa.s.

Fig. 4. Equivalent viscous damping $\zeta_{k}$ versus viscosity of the lubricant meniscus $\eta_{0}$.

Fig. 5. Velocity and ERC time responses and energy decay for contact lubricated with PAO blend 4 ( $\eta_{0}=0.45$ Pa.s) and applied normal force varying from 0.1 to $0.5 \mathrm{~N}$.

Fig. 6. Rate of energy dissipated by velocity dependent friction and by friction independent of the instantaneous sliding velocity versus normal force, for a contact lubricated with PAO blend 4 ( $\eta_{0}=0.45$ Pa.s).

Fig. 7. Evolution of the equivalent viscous damping coefficient $\zeta_{k}$ and friction coefficient $\mu_{k}$ versus applied normal force, for contact lubricated with PAO blends.

Fig. 8. Evolution of the equivalent viscous damping coefficient $\zeta_{k}$ and friction coefficient $\mu_{k}$ versus applied normal force, for contact lubricated with glycerol/water blends or pure glycerol.

Fig. 9. Evolution of the friction coefficient $\mu_{k}$ versus $\eta(P)$ for a contact lubricated with glycerol/water blends, PAO blends and NS base oils.

Fig. 10. Evolution of the friction coefficient $\mu_{k}$ versus dimensionless parameter $\eta(P) /\left(t_{c} . P\right)$ for a contact lubricated with glycerol/water blends, PAO blends and NS base oils. 